\title{
Prognostic value and clinical significance of long noncoding RNA CASC2 in human malignancies: a meta-analysis
}

This article was published in the following Dove Press journal: Cancer Management and Research

\author{
Juan $\mathrm{Cai}^{1}{ }^{1 * *}$ \\ Xueliang Zuo ${ }^{2, *}$ \\ Zhiqiang Chen ${ }^{3,4, *}$ \\ Wenying Zhao' \\ Yiping Zhu' \\ Zhengxiang Zhang' \\ Xiaobing $\mathrm{Ye}^{\mathrm{l}}$ \\ 'Department of Oncology, The First \\ Affiliated Hospital, Yijishan Hospital \\ of Wannan Medical College, Wuhu, \\ Anhui, China; ${ }^{2}$ Department of \\ Gastrointestinal Surgery, The First \\ Affiliated Hospital, Yijishan Hospital \\ of Wannan Medical College, Wuhu, \\ Anhui, China; ${ }^{3}$ Hepatobiliary Center, \\ The First Affiliated Hospital of \\ Nanjing Medical University, Nanjing, \\ Jiangsu, China; ${ }^{4}$ Key Laboratory \\ of Liver Transplantation, Chinese \\ Academy of Medical Sciences, Nanjing, \\ Jiangsu, China \\ *These authors contributed equally to \\ this work
}

Correspondence: Xueliang Zuo Department of Gastrointestinal Surgery, The First Affiliated Hospital, Yijishan Hospital of Wannan Medical College, 2 Zheshan West Road, Jinghu District,

Wuhu 241000, Anhui, China

Tel +865535739558

Email zuoxueliang0202@I26.com

Xiaobing Ye

Department of Oncology, The First

Affiliated Hospital, Yijishan Hospital of

Wannan Medical College, 2 Zheshan

West Road, Jinghu District, Wuhu

241000, Anhui, China

Tel +865535739 I89

E-mail yexbwnmc@I26.com
Purpose: This meta-analysis aimed to assess the prognostic value of long noncoding RNA cancer susceptibility candidate 2 (CASC2) in human tumors.

Materials and methods: We searched the available databases up to December 2017. Pooled hazard ratios (HRs) and the corresponding 95\% confidence intervals (CIs) were used to examine the prognostic impact of CASC2 on overall survival (OS) in patients diagnosed with malignancies.

Results: A total of eight studies with 663 cancer patients were enrolled. Our results showed that high CASC2 expression level was associated with a favorable OS (HR=0.437, 95\% CI: $0.345-0.554)$. The significant results were not altered by stratified analysis according to cancer type, sample size, follow-up months, and HR estimation method. A significant association of glioma tumor stage with CASC2 expression was detected (III-IV vs I-II: odds ratio=2.126, 95\% CI: 1.032-4.378). CASC2 could be used as an independent prognostic factor for OS (HR=0.450, 95\% CI: 0.336-0.602). Sensitivity analysis showed that no single study changed the pooled results significantly. Begg's funnel plot and Egger's test showed that no publication bias was detected.

Conclusion: High expression level of CASC2 is associated with favorable survival outcome for cancer patients, and CASC2 could be used as a prognostic predictor for cancers.

Keywords: CASC2, long noncoding RNA, prognosis, meta-analysis

\section{Introduction}

Cancer is becoming a leading cause of morbidity and mortality around the world. ${ }^{1}$ In 2015, 4,292,000 new cancer cases and 2,814,000 cancer-related deaths occurred in China. ${ }^{2}$ The 5-year survival rate of most cancer patients remains dismal due to lack of effective diagnostic and therapeutic tools. ${ }^{3}$ Previous studies showed that many genes are closely related to cancer progression; however, the complex molecular mechanisms have not been completely elucidated. ${ }^{4,5}$ Therefore, developing a more effective diagnostic and treatment method is an imperative task.

In recent years, increasing evidence has identified that tumorigenesis is associated with long noncoding RNAs (lncRNAs, >200 nucleotides in length). ${ }^{6}$ LncRNAs have been acknowledged as indispensable players in chromatin remodeling, gene regulation, and epigenetic modification at the transcriptional and posttranscriptional levels. ${ }^{7}$ In addition, lncRNAs play an important role in various biological processes, including cell proliferation, differentiation, apoptosis, and invasion. ${ }^{89}$ LncRNAs are less conserved and unable to encode proteins. Given that lncRNAs have been verified as 
crucial molecules in various cancers, ${ }^{10}$ they could be potential biomarkers for cancer prognosis.

Cancer susceptibility candidate 2 (CASC2), located at chromosome 10q26, is an lncRNA that functions as a tumor suppressor. Mounting evidence has demonstrated that CASC2 plays diverse roles in different cancers, including endometrial cancer, cervical cancer, nonsmall cell-lung cancer, renal cell carcinoma, gliomas, ${ }^{11-15}$ and digestive system cancers. ${ }^{16-18}$ Several studies indicated that overexpression of CASC2 inhibits cancer cell proliferation, migration, and invasion. ${ }^{12,14} \mathrm{~A}$ significant decrease in CASC2 expression is associated with poor prognosis of cancer patients. ${ }^{14}$ With a growing number of studies demonstrating the involvement of CASC2 in tumorigenesis, the detection of CASC2 level in cancer patients is valuable for outcome prediction.

However, previous studies reported that the relationship between CASC2 and malignant tumors remains insufficient due to limitations in sample size and discrete outcomes..$^{14,18}$ In view of the aforementioned facts, we comprehensively collected related publications and conducted this study to address the association of CASC 2 expression with patient survival and clinicopathological characteristics. Thus, we analyzed all relevant studies to investigate whether CASC2 could serve as an effective prognostic marker in human cancer.

\section{Materials and methods}

\section{Search strategy}

In order to identify relevant published studies, electronic searches of PubMed, Web of Science, EMBASE, Cochrane Library, Google Scholar, and Chinese National Knowledge Infrastructure database were done for the query "CASC2 or CASC2a" and "cancer or carcinoma or tumor or tumor or neoplasm or malignancy." An additional hand search of references of original or review articles on this topic was performed. The literature search included all relevant studies published until December 2017 for which an abstract or manuscript was available.

\section{Inclusion criteria and exclusion criteria}

Inclusion criteria for the meta-analysis were: 1) the study explored any type of human tumors; 2) the relationship between CASC2 and clinical prognosis was investigated; and 3 ) the study provided usable data for estimating hazard ratios (HRs) and 95\% confidence intervals (CIs) for survival outcomes. The exclusion criteria for articles were: 1) studies without complete data; 2) duplicate publications; and 3) animal studies, case reports, and reviews.

\section{Data extraction}

Titles and abstracts of potentially relevant articles were screened by two independent investigators (CJ and CZQ), and full-text manuscripts meeting the initial screening criteria were obtained. The investigators independently examined all full-text articles for inclusion in the review; any discrepancies were discussed by the investigators. If they were unable to reach consensus, a third investigator (ZXL) was consulted. Standardized data extraction tables were created, and data extraction was completed by CZQ and checked by ZXL for accuracy. Information including the first author's name, year of publication, cancer type, tumor stage, sample size, cutoff value, CASC2 detection method, preoperative treatment, follow-up months, survival analysis, and HRs with the corresponding 95\% CIs was extracted. If HRs and their $95 \%$ CIs could not be obtained directly from publications, they were extracted from Kaplan-Meier curves by using the Engauge Digitizer Version 4.1 (http://markummitchell.github. io/engauge-digitizer/). ${ }^{19}$ The quality of the literature was evaluated according to the Newcastle-Ottawa Scale (NOS) ${ }^{20}$ and the studies with NOS score $\geq 7$ were considered to be high quality.

\section{Statistical analysis}

All meta-analyses were performed with Stata software version 12.0 (StataCorp LP, College Station, TX, USA). HRs with the corresponding $95 \%$ CIs were used to estimate the strength of the relationship between CASC2 and clinical prognosis of the cancer patients. Pooled odds ratios (ORs) with $95 \%$ CIs were used to assess the association of IncRNA expression with clinicopathological characteristics. Heterogeneity between studies was quantified by chi-squared test and $I^{2}$ statistics. A chi-squared test of $p<0.10$ or $I^{2}>50 \%$ indicated heterogeneity across the studies. If the heterogeneity was not significant, we used a fixed effect model to investigate the pooled HRs. Random effect model was used to summarize the statistical synthesis if between-study heterogeneity was substantial. Subgroup analyses were conducted according to cancer type, sample size, follow-up months, and HR estimation method. Sensitivity analysis was performed by sequentially omitting individual study to guarantee the robustness of the results. Begg's funnel plot and Egger's test were employed to evaluate the risk of publication bias. $p<0.05$ was considered as statistically significant.

\section{Results}

\section{Study characteristics}

The search strategy retrieved 328 potentially relevant studies. Based on the screening criteria, eight eligible studies with 663 patients were enrolled in the current meta-analysis. ${ }^{14,18,21-26}$ 
The flowchart of this study selection was concluded and shown in Figure 1. Study characteristics of these eight studies were summarized in Table 1. All studies were published in recent 2 years, suggesting that the prognostic value of CASC2 is a novel field of research. Of the enrolled studies, three focused on digestive system malignancies, including pancreatic cancer, gastric cancer, and hepatocellular carcinoma (HCC), while the other five studies on nondigestive system malignancies (thyroid carcinoma, glioma, and lung cancer). Eight studies were for overall survival (OS) and one for disease-free survival. The maximum and minimum sample size were 110 and 57, respectively. The follow-up time ranged between 32 and 70 months.

\section{Association of CASC2 with OS}

A total of eight studies assessed the HRs for OS. No heterogeneity was found among the studies (chi-squared $=6.26, \mathrm{df}=7$, $p=0.509, I^{2}=0 \%$ ), thus a fixed effect model was employed to pool the association of CASC2 expression with OS of multiple malignancies. As illustrated in Figure 2 and Table 2, it was indicated that increased CASC2 expression predicted a favorable outcome for OS of cancer patients (pooled $\mathrm{HR}=0.437,95 \% \mathrm{CI}: 0.345-0.554)$.

Subsequent stratified analyses were performed according to cancer type, sample size, follow-up months, and HR estimation method (Figure 3A-D and Table 2). A significant relationship between CASC2 overexpression and a favorable OS was observed in patients with digestive system cancer $(\mathrm{HR}=0.399,95 \% \mathrm{CI}: 0.275-0.580)$ and nondigestive system cancer patients ( $\mathrm{HR}=0.464,95 \% \mathrm{CI}: 0.343-0.630)$. In the analysis stratified by sample size, CASC2 was found to be significantly correlated to patient survival in studies with sample size less than the median value of $74(\mathrm{HR}=0.410$, 95\% CI: $0.296-0.568)$ and $>74(\mathrm{HR}=0.470,95 \% \mathrm{CI}$ : 0.333-0.661). There was a significant correlation between CASC2 expression level and patient survival outcome in studies with follow-up time $>60$ months $(\mathrm{HR}=0.448,95 \%$ CI: $0.322-0.623)$ and $<60$ months $(\mathrm{HR}=0.426,95 \% \mathrm{CI}$ : $0.304-0.597)$. In addition, stratified analysis on HR estimation method revealed a statistical significance in both the subgroups (indirectly: $\mathrm{HR}=0.414,95 \% \mathrm{CI}$ : 0.277-0.620; directly: HR=0.450, 95\% CI: 0.336-0.602).
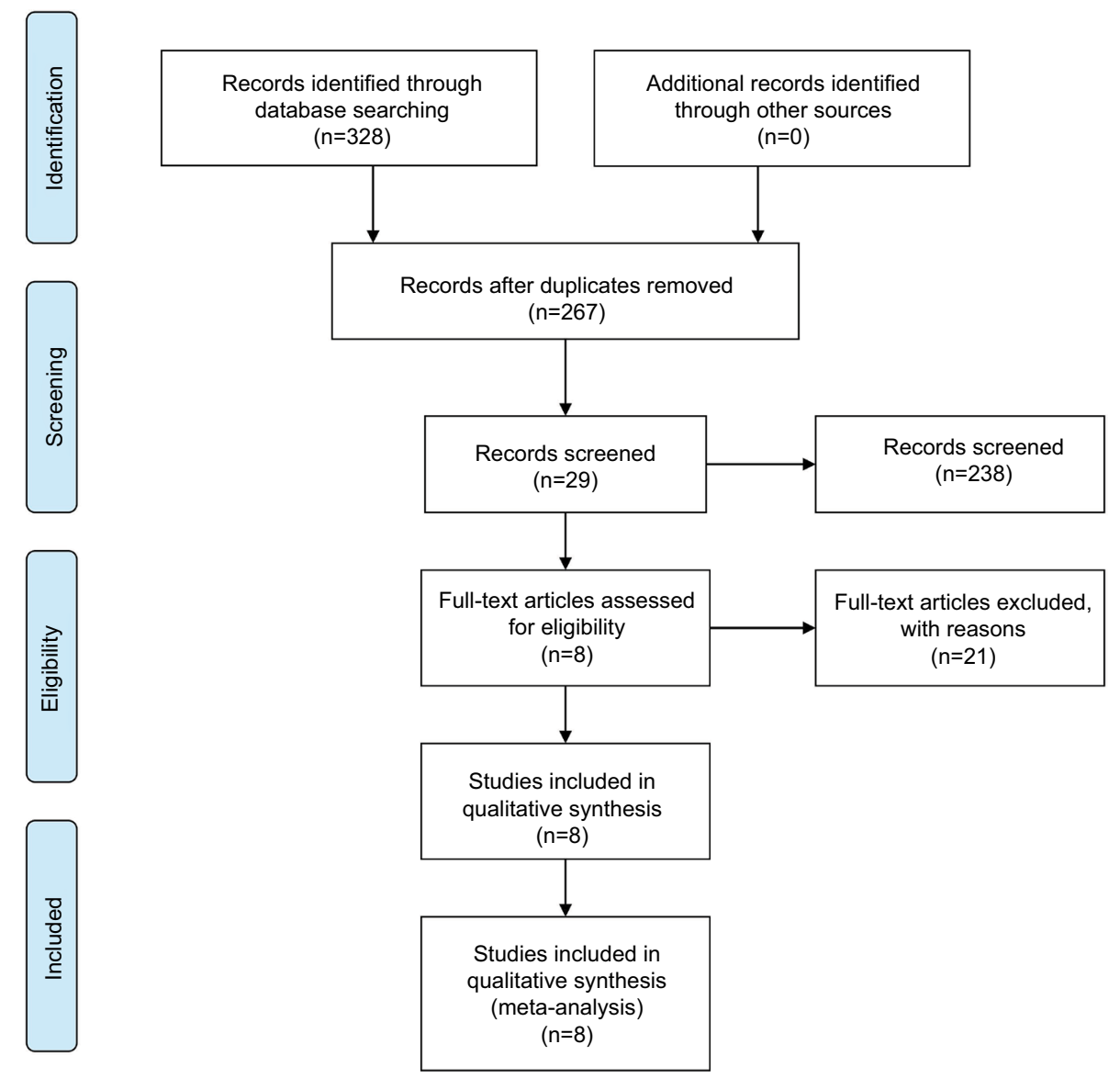

Figure I Flowchart of the study search and selection in this meta-analysis. 


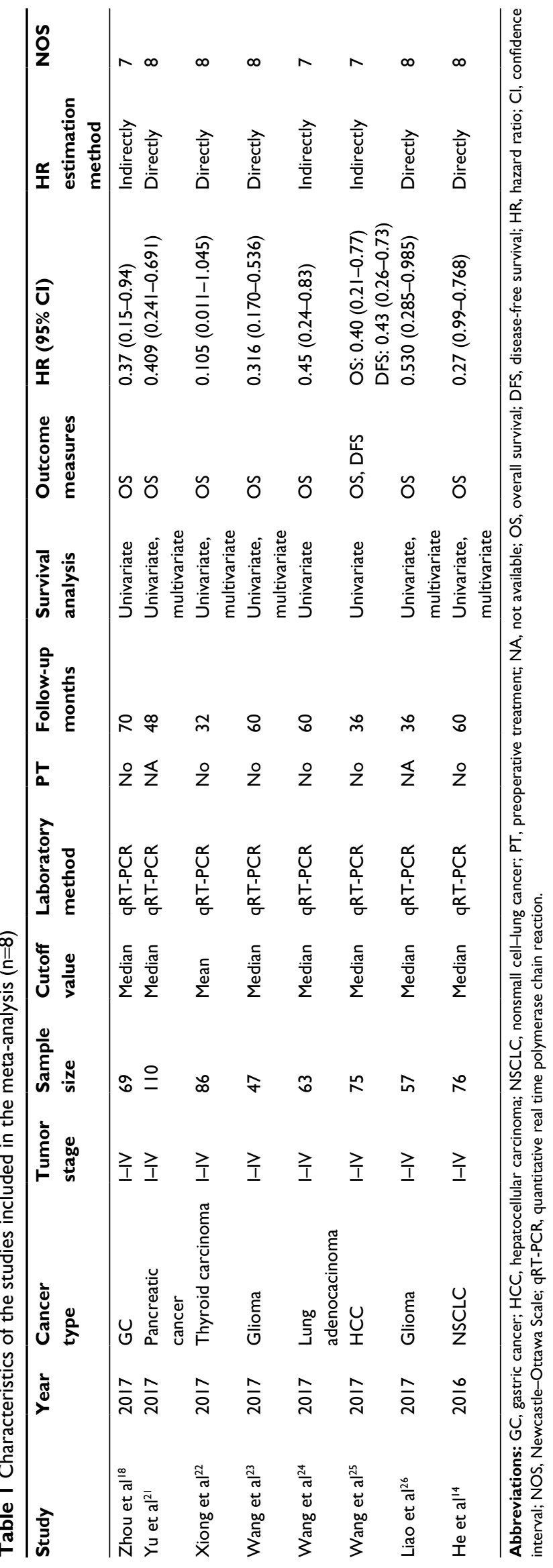

To investigate the robustness of our pooled estimates, we performed sensitivity analysis by omitting one study each time. The sensitivity analysis showed that no individual study altered the pooled results significantly, confirming the stability of our data (Figure 4).

\section{Independent prognostic value of CASC2}

Cox proportional multivariate model was used in five studies to assess the role of CASC2 as an independent prognostic factor for various malignancies. As shown in Figure 5, it was revealed that CASC2 was an independent prognostic factor for OS in tumor patients (pooled $\mathrm{HR}=0.450,95 \% \mathrm{CI}$ : $0.336-0.602$ ). No statistically significant heterogeneity was found across these studies (chi-squared $=6.02, \mathrm{df}=4, p=0.198$, $\left.r^{2}=33.6 \%\right)$.

\section{Correlation between CASC2 and clinicopathological features}

A total of six studies investigated the correlation between CASC2 expression and clinicopathological features of human tumors. Two studies focused on glioma, two on HCC, and two on lung cancer. A significant association of glioma tumor stage with CASC2 expression was detected (III-IV vs I-II: $\mathrm{OR}=2.126$, 95\% CI: $1.032-4.378$ ). However, the pooled estimates showed that CASC2 expression was not correlated to other clinicopathological features in glioma, HCC, and lung cancer (Table 3).

\section{Publication bias}

Begg's funnel plot and Egger's test were used to assess the potential bias in the available literature. The shape of funnel plots showed no evidence of asymmetry (Figure 6). In addition, Egger's test indicated that there was no significant publication bias $(p=0.332)$.

\section{Discussion}

Recent genome studies have demonstrated that dysfunctional IncRNAs are involved in tumor occurrence and progression. ${ }^{27,28}$ Considered as novel molecular biomarkers, lncRNAs may be used for the prediction of cancer prognosis. Previous studies have shown that CASC2 expression level is downregulated in many cancers, including nonsmall cell-lung cancer, ${ }^{24}$ gliomas, ${ }^{15}$ gynecological malignant tumor, ${ }^{13}$ urinary system cancers, ${ }^{29,30}$ and digestive tract tumors. ${ }^{16,21}$ To assess the prognostic role of CASC2 in various human cancers, we implemented this comprehensive meta-analysis. Our statistically significant results of pooled estimates suggested that CASC2 could be used as a prognostic candidate for most human malignancies. 
Study

ID

$$
\text { HR }(95 \% \mathrm{Cl}) \quad \text { Weight }
$$

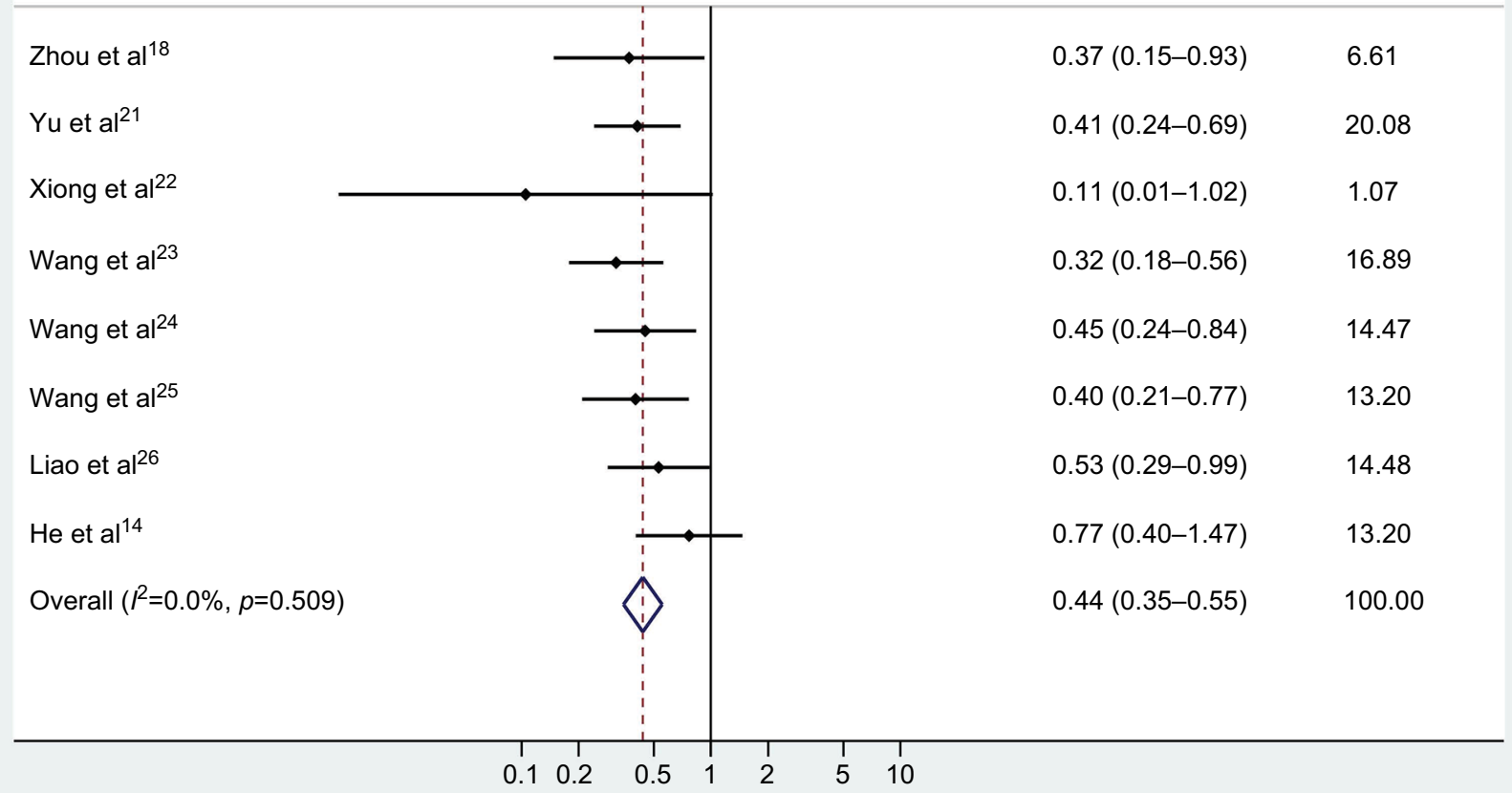

Figure 2 Forest plot for the association of CASC2 expression with overall survival of human malignancies.

Abbreviations: $\mathrm{HR}$, hazard ratio; $\mathrm{Cl}$, confidence interval; $\mathrm{CASC} 2$, cancer susceptibility candidate 2 .

Table 2 Results of subgroup analysis of the pooled HRs of overall survival of patients with increased long noncoding CASC2 expression

\begin{tabular}{|c|c|c|c|c|c|}
\hline \multirow[t]{2}{*}{ Subgroup analysis } & \multirow{2}{*}{$\begin{array}{l}\text { No. of } \\
\text { studies }\end{array}$} & \multirow{2}{*}{$\begin{array}{l}\text { No. of } \\
\text { patients }\end{array}$} & \multirow[t]{2}{*}{ Pooled HR (95\% CI) } & \multicolumn{2}{|c|}{ Heterogeneity } \\
\hline & & & & $I^{2}(\%)$ & $p$-value \\
\hline Total & 8 & 663 & $0.437(0.345-0.554)$ & 0 & 0.509 \\
\hline \multicolumn{6}{|l|}{ Cancer type } \\
\hline Digestive system cancer & 3 & 254 & $0.399(0.275-0.580)$ & 0 & 0.983 \\
\hline Nondigestive system cancer & 5 & 409 & $0.464(0.343-0.630)$ & 31.7 & 0.210 \\
\hline \multicolumn{6}{|l|}{ Sample size } \\
\hline Number $<74$ & 4 & 236 & $0.410(0.296-0.568)$ & 0 & 0.663 \\
\hline Number $\geq 74$ & 4 & 427 & $0.470(0.333-0.661)$ & 31.3 & 0.225 \\
\hline \multicolumn{6}{|l|}{ Follow-up months } \\
\hline$\geq 60$ & 4 & 255 & $0.448(0.322-0.623)$ & 29.1 & 0.238 \\
\hline$<60$ & 4 & 408 & $0.426(0.304-0.597)$ & 0 & 0.575 \\
\hline \multicolumn{6}{|l|}{ HR estimation method } \\
\hline Indirectly & 3 & 207 & $0.4 \mid 4(0.277-0.620)$ & 0 & 0.933 \\
\hline Directly & 5 & 456 & $0.450(0.336-0.602)$ & 33.6 & 0.198 \\
\hline
\end{tabular}

Abbreviations: $\mathrm{HR}$, hazard ratio; $\mathrm{Cl}$, confidence interval; $\mathrm{CASC}$, cancer susceptibility candidate 2 .

Systematic analysis of the relationship between CASC2 and cancer prognosis has not been reported yet. Therefore, we included eight eligible studies assessing the prognostic value of CASC2. A total of 663 individuals were enrolled in this meta-analysis. A significant correlation between CASC2 overexpression and a favorable OS was observed, suggesting that CASC2 may be used as a predictor for OS of cancer patients. Subgroup analyses, including tumor type, sample size, follow-up time, and HR estimation method, showed that the value of CASC2 for OS existed in all subgroups. Cox 
A

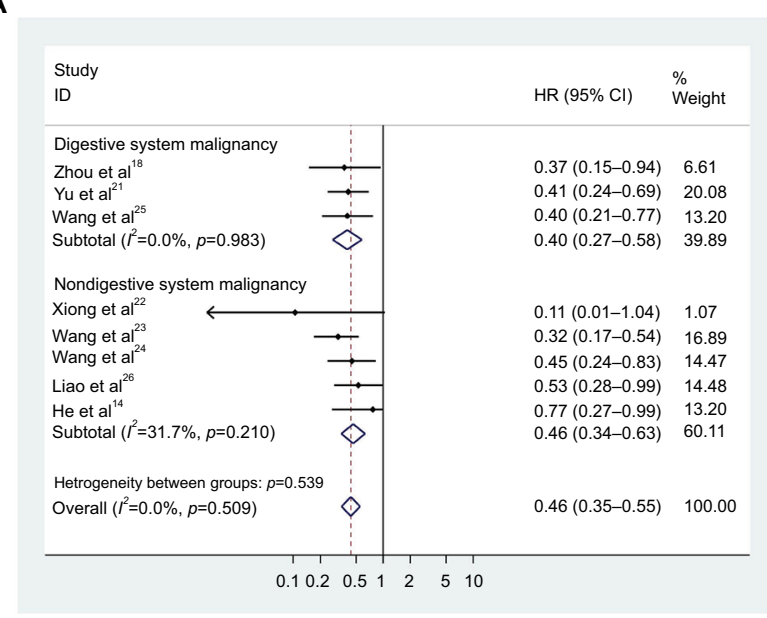

C

\begin{tabular}{|c|c|c|c|}
\hline $\begin{array}{l}\text { Study } \\
\text { ID }\end{array}$ & & $\mathrm{HR}(95 \% \mathrm{Cl})$ & $\begin{array}{l}\% \\
\text { Weight }\end{array}$ \\
\hline \multicolumn{4}{|l|}{ Follow-up months $\geq 60$} \\
\hline Zhou et al ${ }^{18}$ & & $0.37(0.15-0.94)$ & 6.61 \\
\hline Wang et $\mathrm{al}^{23}$ & $\rightarrow$ & $0.32(0.17-0.54)$ & 16.89 \\
\hline Wang et $\mathrm{al}^{24}$ & & $0.45(0.24-0.83)$ & 14.47 \\
\hline He et $\mathrm{ll}^{14}$ & & $0.77(0.27-0.99)$ & 13.20 \\
\hline Subtotal $\left(I^{2}=29.1 \%, p=0.238\right)$ & 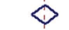 & $0.45(0.32-0.62)$ & 51.17 \\
\hline \multicolumn{4}{|l|}{ Follow-up months $<60$} \\
\hline Yu et $\mathrm{al}^{21}$ & - & $0.45(0.24-0.69)$ & 20.08 \\
\hline Xiong et al ${ }^{22}$ & & $0.11(0.01-1.04)$ & 1.07 \\
\hline Wang et $\mathrm{al}^{25}$ & $\rightarrow$ & $0.40(0.21-0.77)$ & 13.20 \\
\hline Liao et $\mathrm{al}^{26}$ & + & $0.53(0.28-0.99)$ & 14.48 \\
\hline Subtotal $\left(I^{2}=0.0 \%, p=0.575\right)$ & $>$ & $0.43(0.30-0.60)$ & 48.83 \\
\hline \multicolumn{4}{|c|}{ Heterogeneity between groups: $p=0.834$} \\
\hline Overall $\left(I^{2}=0.0 \%, p=0.509\right)$ & $\diamond$ & $0.44(0.35-0.55)$ & 100.00 \\
\hline
\end{tabular}

B

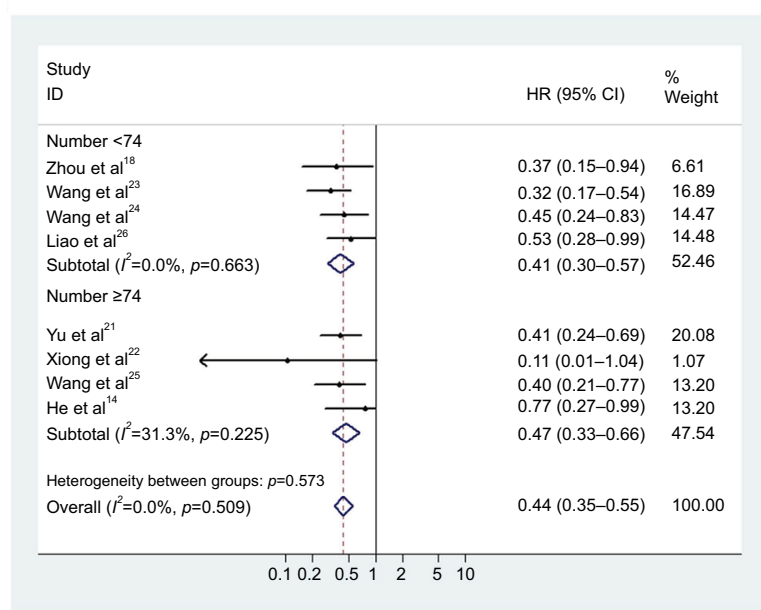

D

\begin{tabular}{ll|llll} 
Study \\
ID
\end{tabular}

Figure 3 Forest plots of the subgroup analyses evaluating HRs of CASC2 for overall survival by the factors of cancer type (A), sample size (B), follow-up months (C), and HR estimation method (D).

Abbreviations: $\mathrm{HR}$, hazard ratio; $\mathrm{Cl}$, confidence interval; CASC2, cancer susceptibility candidate 2 .

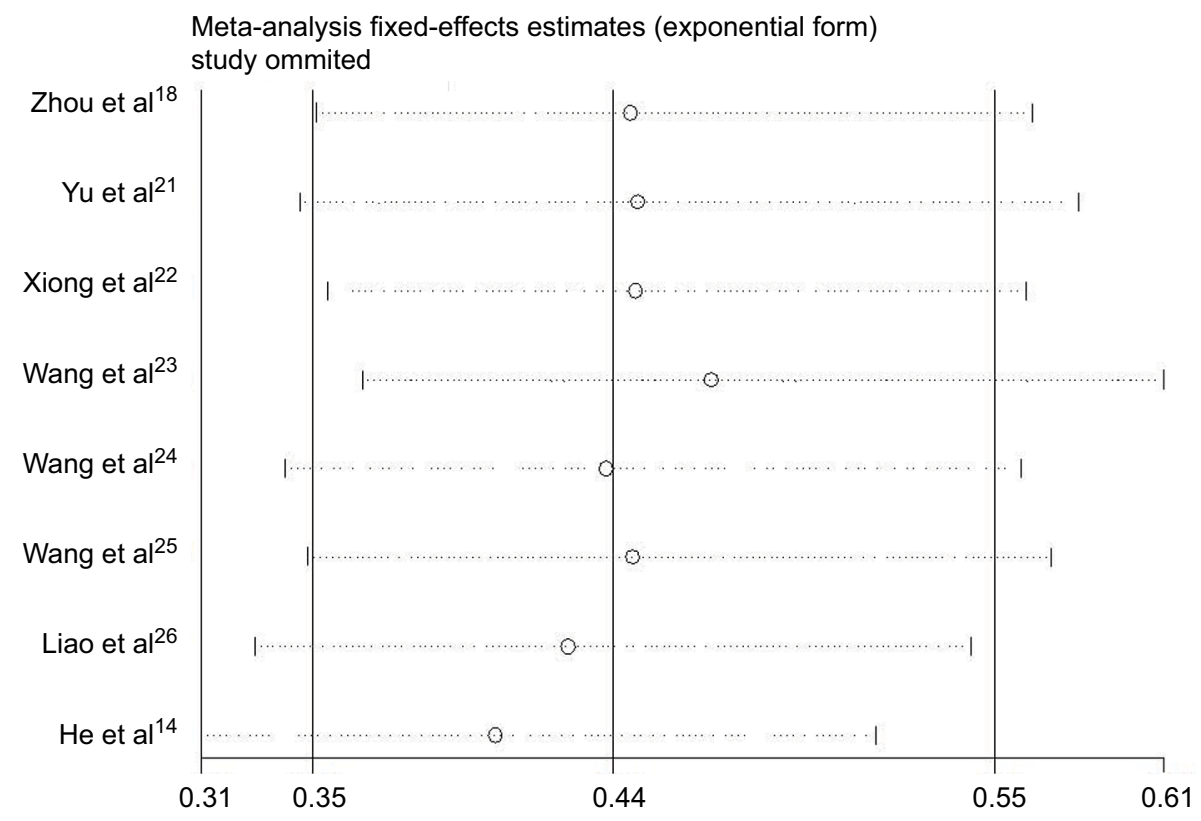

Figure 4 Sensitivity analysis of the effect of the individual study on the pooled HRs for the correlation between CASC2 and overall survival. Abbreviations: $\mathrm{HR}$, hazard ratio; $\mathrm{Cl}$, confidence interval; CASC2, cancer susceptibility candidate 2. 
Study

ID $\begin{array}{ll}\% & \% \\ H R(95 \% \mathrm{Cl}) & \text { Weight }\end{array}$

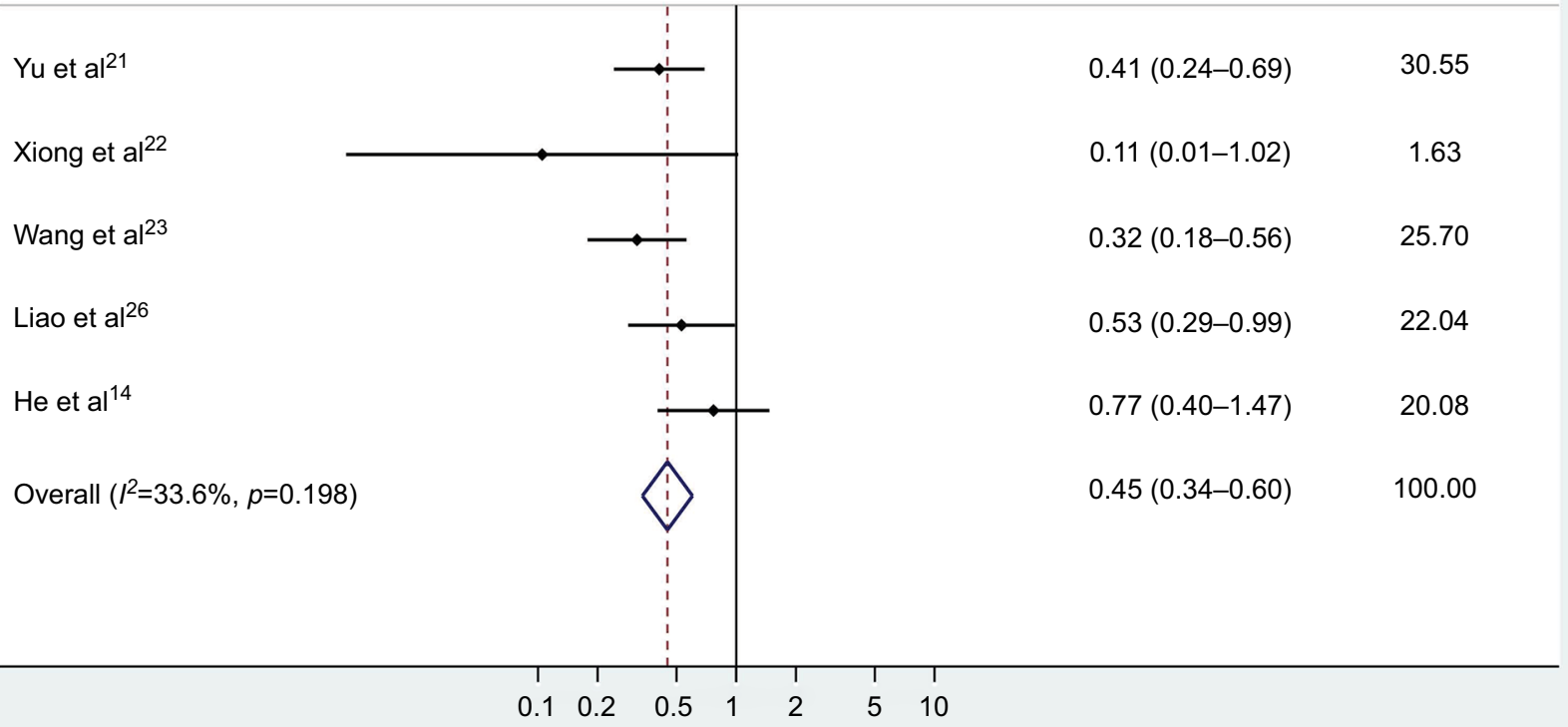

Figure 5 Meta-analysis of the independent role of CASC2 in overall survival.

Abbreviations: $\mathrm{HR}$, hazard ratio; $\mathrm{Cl}$, confidence interval; CASC2, cancer susceptibility candidate 2.

Table 3 Results of the association of CASC2 expression with clinicopathological features

\begin{tabular}{|c|c|c|c|c|c|}
\hline \multirow[t]{2}{*}{ Clinicopathological features } & \multirow{2}{*}{$\begin{array}{l}\text { No. of } \\
\text { studies }\end{array}$} & \multirow{2}{*}{$\begin{array}{l}\text { Pooled OR } \\
(95 \% \mathrm{CI})\end{array}$} & \multirow[t]{2}{*}{$p$-value } & \multicolumn{2}{|c|}{ Heterogeneity } \\
\hline & & & & $I^{2}(\%)$ & $p$-value \\
\hline \multicolumn{6}{|l|}{ Glioma } \\
\hline Age ( $\geq 45$ vs $<45$ years) & 2 & $0.813(0.425-1.555)$ & 0.797 & 0 & 0.531 \\
\hline Gender (male vs female) & 2 & $1.059(0.558-2.011)$ & 0.712 & 0 & 0.860 \\
\hline Tumor stage (III-IV vs I-II) & 2 & $2.126(1.032-4.378)$ & 0.041 & 0 & 0.936 \\
\hline \multicolumn{6}{|l|}{$\mathrm{HCC}$} \\
\hline Age ( $\geq 50$ vs $<50$ years) & 2 & $1.023(0.579-1.808)$ & 0.938 & 0 & 0.843 \\
\hline Gender (male vs female) & 2 & $0.940(0.557-1.587)$ & 0.816 & 0 & 0.864 \\
\hline Tumor number (multiple vs single) & 2 & $1.453(0.574-3.679)$ & 0.431 & 22.40 & 0.257 \\
\hline Tumor size $(\geq 5$ vs $<5 \mathrm{~cm})$ & 2 & $1.394(0.755-2.574)$ & 0.288 & 0 & 0.869 \\
\hline TNM stage (III-IV vs I-II) & 2 & $1.955(0.58 \mathrm{I}-6.576)$ & 0.279 & 61.40 & 0.107 \\
\hline $\operatorname{AFP}(\mathrm{ng} / \mathrm{mL})(\geq 400$ vs $<400)$ & 2 & $1.319(0.716-2.431)$ & 0.374 & 0 & 0.394 \\
\hline HBV (yes vs no) & 2 & $1.059(0.622-1.804)$ & 0.832 & 0 & 0.640 \\
\hline \multicolumn{6}{|l|}{ Lung cancer } \\
\hline Gender (male vs female) & 2 & $1.017(0.559-1.849)$ & 0.956 & 0 & 0.485 \\
\hline TNM stage (III-IV vs I-II) & 2 & $1.881(0.960-3.688)$ & 0.066 & 0 & 0.873 \\
\hline Lymph node metastasis (positive vs negative) & 2 & $1.300(0.740-2.285)$ & 0.361 & 0 & 0.541 \\
\hline Tumor size $(\geq 3$ vs $<3 \mathrm{~cm})$ & 2 & $1.660(0.939-2.935)$ & 0.081 & 0 & 0.450 \\
\hline
\end{tabular}

Abbreviations: OR, odds ratio; $\mathrm{Cl}$, confidence interval; $\mathrm{HCC}$, hepatocellular carcinoma; TNM, tumor-node metastasis; AFP, $\alpha$-fetoprotein; HBV, hepatitis B virus; CASC2, cancer susceptibility candidate 2 . 


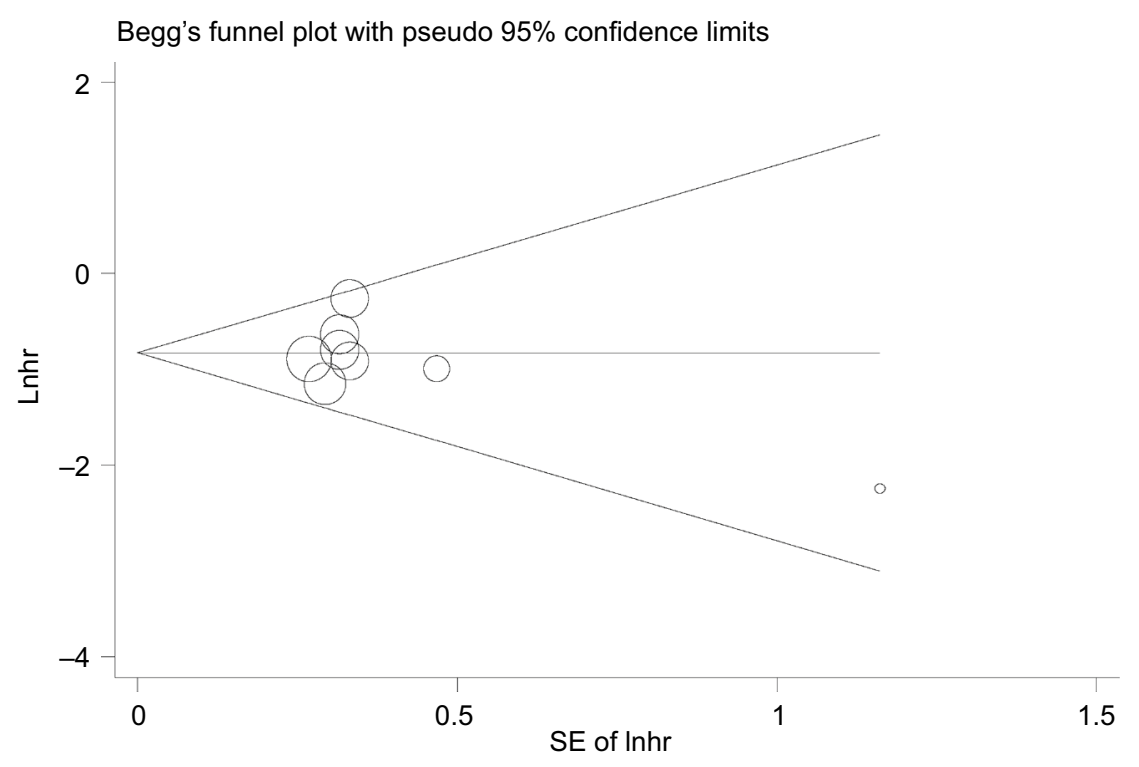

Figure 6 Begg's funnel plot of publication bias.

Abbreviations: SE, standard error; Lnhr, natural logarithm of hazards ratio.

proportional multivariate model indicated that CASC2 was an independent prognostic factor for OS in cancer patients. Furthermore, we found that advanced tumor stage was associated with downregulated CACS2 expression in glioma. Our data also indicated that there was no correlation between CASC2 and other clinicopathological features, including age, gender, tumor size, and lymph node metastasis. Begg's funnel plot and Egger's test revealed no significant publication bias. Further sensitivity analysis suggested that our results were robust.

CASC2 is a novel lncRNA involved in cancer progression and correlated to tumor prognosis. Three different mRNAs, CASC2a, CASC2b, and CASC2c, originated from alternative splicing of the five exons of CASC2. ${ }^{11}$ Previous studies have indicated that $\mathrm{CASC} 2 \mathrm{a}$ was downregulated in cancer tissue specimens and cell lines. ${ }^{18,21}$ Most studies focused on CASC2a, whereas Liu et $\mathrm{al}^{31}$ investigated the function of CASC2c. The present meta-analysis evaluated the prognostic value of CASC2a in human malignancies; therefore, we ruled out the study on CASC2c from our analysis.

A recent review has summarized the potential functions and pathways associated with CASC2. ${ }^{32}$ The study has shown that CASC2 regulates multiple key regulators in cancer-associated signaling transduction network and acts as a powerful tumor suppressor. By sponging a panel of microRNAs or other elements, CASC2 modulates several important signaling pathways, including the PI3K/Akt, NK- $\mathrm{BB}, \mathrm{MAPK}$, Wnt/ $\beta$-catenin, and Jak/Stat3 signaling. Cao et $\mathrm{al}^{29}$ reported that the expression level of CASC2 in renal cell carcinoma is significantly lower than that in normal tissue. Regulated by
miR-21, CASC2 suppresses the development of renal cell carcinoma cells. In colorectal cancer, CASC2 increases protein inhibitor of activated STAT 3 expression level by functioning as a competing endogenous RNA for miR-18a, resulting in enhanced cancer cell proliferation. ${ }^{6} \mathrm{CASC} 2$ regulates MAPK signaling pathway to suppress gastric cancer ${ }^{33}$ and $\mathrm{HCC}^{34}$ cells in vivo and in vitro. Moreover, CASC2a is associated with the risk of recurrence among patients undergoing radical cystectomy, indicating that CASC 2 a could act as a survival predictor and potential therapeutic target in bladder cancer. ${ }^{35}$ It has also been reported that overexpression of CASC2 reduces bladder cancer cell growth via suppressing Wnt/ $\beta$-catenin pathway. ${ }^{30}$ In addition, CASC2 increases the sensitivity of chemotherapeutic drugs, such as cisplatin and docetaxel. CASC2 overexpression could sensitize cisplatinresistant cervical cancer cell to cisplatin through targeting miR-21 and miR-181a and inhibiting PI3K/Akt pathway. ${ }^{13,26}$ Gao et $\mathrm{al}^{36}$ reported that CASC2 competes with SPRY2 for miR-183 binding to enhance the sensitivity of prostate cancer cells to docetaxel via SPRY2 downstream ERK signaling pathway. Hence, CASC 2 could exert a strong antitumor effect and function as a promising prognostic factor for patients diagnosed with malignancies.

Despite our efforts to make a comprehensive study, this meta-analysis still has several limitations. First, the sample size is relatively small. Given the limited number of literature pertaining to the prognostic role of CASC 2 in a single type of tumor, we were unable to pool the results in one type of malignancy. To handle this issue, we attempted to analyze the role of CASC2 in digestive system and nondigestive 
system cancers and confirmed the prognostic significance of CASC2 in both the subgroups. Second, the prognostic value of CASC2 is a relatively novel field of research, and the included studies were published in recent 2 years. All enrolled patients were from China, which means our results might not be applied to different ethnicities and regions. Therefore, more large-scale studies are duly warranted to further verify the prognostic value of CASC2 in different ethnicities. Third, the cutoff value for CASC2 expression varied across different studies, since a consensus value was hard to reach. Fourth, some of the HRs were extracted indirectly through reconstructing Kaplan-Meier survival curves. Fifth, the data collection may be incomplete because we only retrieve literature from Chinese and English databases. Therefore, it might be possible that our meta-analysis overestimated the predictive significance of $\mathrm{CASC} 2$ in cancer patients.

\section{Conclusion}

In summary, our results showed that downregulation of CASC2 expression is associated with poor OS in various cancers. Accordingly, CASC 2 expression might be used as a novel biomarker to predict the prognosis of cancer patients. Thus, more clinical studies investigating more types of cancer with larger sample size are needed to further clarify the role of CASC2 in cancer prognosis.

\section{Acknowledgment}

This study was funded by the Natural Science Research of Anhui Education Department Key Project (No. KJ2018A0246).

\section{Disclosure}

The authors report no conflicts of interest in this work.

\section{References}

1. Torre LA, Bray F, Siegel RL, Ferlay J, Lortet-Tieulent J, Jemal A. Global cancer statistics, 2012. CA Cancer J Clin. 2015;65(2):87-108.

2. Chen W, Zheng R, Baade PD, et al. Cancer statistics in China, 2015. CA Cancer J Clin. 2016;66(2):115-132.

3. Liotta LA, Ferrari M, Petricoin E. Clinical proteomics: written in blood. Nature. 2003;425(6961):905.

4. Wang Y, Huang Y, Xiang P, Tian W. LncRNA expression and implication in osteosarcoma: a systematic review and meta-analysis. Onco Targets Ther. 2017;10:5355-5361.

5. Zhang $\mathrm{C}$, Liu $\mathrm{C}, \mathrm{Wu} \mathrm{J}$, et al. Upregulation of long noncoding RNA LOC440040 promotes tumor progression and predicts poor prognosis in patients with prostate cancer. Onco Targets Ther. 2017;10:4945-4954.

6. Huang G, Wu X, Li S, Xu X, Zhu H, Chen X. The long noncoding RNA CASC2 functions as a competing endogenous RNA by sponging miR-18a in colorectal cancer. Sci Rep. 2016;6:26524.

7. Takahashi K, Yan I, Haga H, Patel T. Long noncoding RNA in liver diseases. Hepatology. 2014;60(2):744-753.
8. Geisler S, Coller J. RNA in unexpected places: long non-coding RNA functions in diverse cellular contexts. Nat Rev Mol Cell Biol. 2013;14(11):699-712.

9. Gutschner T, Diederichs S. The hallmarks of cancer: a long non-coding RNA point of view. RNA Biol. 2012;9(6):703-719.

10. Gupta RA, Shah N, Wang KC, et al. Long non-coding RNA HOTAIR reprograms chromatin state to promote cancer metastasis. Nature. 2010;464(7291):1071-1076.

11. Baldinu P, Cossu A, Manca A, et al. Identification of a novel candidate gene, CASC2, in a region of common allelic loss at chromosome 10q26 in human endometrial cancer. Hum Mutat. 2004;23(4):318-326.

12. Baldinu P, Cossu A, Manca A, et al. CASC2a gene is down-regulated in endometrial cancer. Anticancer Res. 2007;27(1A):235-243.

13. Feng Y, Zou W, Hu C, et al. Modulation of CASC2/miR-21/PTEN pathway sensitizes cervical cancer to cisplatin. Arch Biochem Biophys. 2017;623-624:20-30.

14. He X, Liu Z, Su J, et al. Low expression of long noncoding RNA CASC2 indicates a poor prognosis and regulates cell proliferation in non-small cell lung cancer. Tumour Biol. 2016;37(7):9503-9510.

15. Jiang C, Shen F, Du J, et al. Upregulation of CASC2 sensitized glioma to temozolomide cytotoxicity through autophagy inhibition by sponging miR-193a-5p and regulating mTOR expression. Biomed Pharmacother. 2017;97:844-850.

16. Zhang W, He W, Gao J, et al. The long noncoding RNA CASC2 inhibits tumorigenesis through modulating the expression of PTEN by targeting miR-18a-5p in esophageal carcinoma. Exp Cell Res. 2017;361(1):30-38.

17. Zeng F, Le YG, Fan JC, Xin L. LncRNA CASC2 inhibited the viability and induced the apoptosis of hepatocellular carcinoma cells through regulating miR-24-3p. J Cell Biochem. 2017 Epub Nov 1.

18. Zhou J, Huang H, Tong S, Huo R. Overexpression of long non-coding RNA cancer susceptibility 2 inhibits cell invasion and angiogenesis in gastric cancer. Mol Med Rep. 2017;16(4):5235-5240.

19. Tierney JF, Stewart LA, Ghersi D, Burdett S, Sydes MR. Practical methods for incorporating summary time-to-event data into meta-analysis. Trials. 2007;8:16.

20. Stang A. Critical evaluation of the Newcastle-Ottawa scale for the assessment of the quality of nonrandomized studies in meta-analyses. Eur J Epidemiol. 2010;25(9):603-605.

21. Yu Y, Liang S, Zhou Y, Li S, Li Y, Liao W. HNF1A/CASC2 regulates pancreatic cancer cell proliferation through PTEN/Akt signaling. J Cell Biochem. 2017 Epub Sep 2.

22. Xiong $\mathrm{X}$, Zhu $\mathrm{H}$, Chen $\mathrm{X}$. Low expression of long noncoding RNA CASC2 indicates a poor prognosis and promotes tumorigenesis in thyroid carcinoma. Biomed Pharmacother. 2017;93:391-397.

23. Wang P, Liu YH, Yao YL, et al. Long non-coding RNA CASC2 suppresses malignancy in human gliomas by miR-21. Cell Signal. 2015;27(2):275-282.

24. Wang D, Gao ZM, Han LG, Xu F, Liu K, Shen Y. Long noncoding RNA CASC2 inhibits metastasis and epithelial to mesenchymal transition of lung adenocarcinoma via suppressing SOX4. Eur Rev Med Pharmacol Sci. 2017;21(20):4584-4590.

25. Wang Y, Liu Z, Yao B, et al. Long non-coding RNA CASC2 suppresses epithelial-mesenchymal transition of hepatocellular carcinoma cells through CASC2/miR-367/FBXW7 axis. Mol Cancer. 2017;16(1):123.

26. Liao Y, Shen L, Zhao H, et al. LncRNA CASC2 interacts with miR181 a to modulate glioma growth and resistance to TMZ through PTEN pathway. J Cell Biochem. 2017;118(7):1889-1899.

27. Xu X, Xu Y, Shi C, et al. A genome-wide comprehensively analyses of long noncoding RNA profiling and metastasis associated lncRNAs in renal cell carcinoma. Oncotarget. 2017;8(50):87773-87781.

28. Mitra R, Chen X, Greenawalt EJ, et al. Decoding critical long noncoding RNA in ovarian cancer epithelial-to-mesenchymal transition. Nat Commun. 2017;8(1):1604.

29. Cao Y, Xu R, Xu X, Zhou Y, Cui L, He X. Downregulation of IncRNA CASC2 by microRNA-21 increases the proliferation and migration of renal cell carcinoma cells. Mol Med Rep. 2016;14(1):1019-1025. 
30. Pei Z, Du X, Song Y, et al. Down-regulation of 1ncRNA CASC2 promotes cell proliferation and metastasis of bladder cancer by activation of the Wnt/beta-catenin signaling pathway. Oncotarget. 2017;8(11): 18145-18153.

31. Liu C, Sun Y, She X, et al. CASC2c as an unfavorable prognosis factor interacts with miR-101 to mediate astrocytoma tumorigenesis. Cell Death Dis. 2017;8(3):e2639.

32. Palmieri G, Paliogiannis P, Sini MC, et al. Long non-coding RNA CASC2 in human cancer. Crit Rev Oncol Hematol. 2017;111:31-38.

33. Li P, Xue WJ, Feng Y, Mao QS. Long non-coding RNA CASC2 suppresses the proliferation of gastric cancer cells by regulating the MAPK signaling pathway. Am J Transl Res. 2016;8(8):3522-3529.
34. Gan YY, Han NN, He XQ, et al. Long non-coding RNA CASC2 regulates cell biological behaviour through the MAPK signalling pathway in hepatocellular carcinoma. Tumor Biol. 2017;39(6): 1010428317706229.

35. Li Z, Wang KE, Zhou XL, Zhou J, Ye CH. Long non-coding RNA cancer susceptibility candidate $2 \mathrm{a}(\mathrm{CASC} 2 \mathrm{a})$ is a marker of early recurrence after radical cystectomy in patients with urothelial carcinoma of the bladder. Med Sci Monit. 2018;24:438-447.

36. Gao W, Lin S, Cheng C, et al. Long non-coding RNA CASC2 regulates Sprouty2 via functioning as a competing endogenous RNA for miR-183 to modulate the sensitivity of prostate cancer cells to docetaxel. Arch Biochem Biophys. 2018Epub Jan 23.

\section{Publish your work in this journal}

Cancer Management and Research is an international, peer-reviewed open access journal focusing on cancer research and the optimal use of preventative and integrated treatment interventions to achieve improved outcomes, enhanced survival and quality of life for the cancer patient. The manuscript management system is completely online and includes a very quick and fair peer-review system, which is all easy to use. Visit http://www.dovepress.com/testimonials.php to read real quotes from published authors. 